\title{
Plasma treatment of gelatin photography
}

\author{
Blanka Zachariášová, Katarína Haberová, \\ Michal Oravec, Viera Jančovičová \\ Department of Graphic Arts Technology and Applied Photochemistry, \\ Faculty of Chemical and Food Technology, Slovak University of Technology in Bratislava, \\ Radlinského 9, 81237 Bratislava, Slovakia \\ blanka.zachariasova@gmail.com
}

\begin{abstract}
Plasma pre-treatment represents the key enabler technology for microfine cleaning, surface activation and plasma coating of almost all types of materials - from plastics, metals and glasses to textiles, composites and photographs. More and more conventional industrial treatment methods are being replaced by plasma technology in order to make processes more effective and environmentally friendly. This study is oriented on the characterization of a photographic image on two types of photographic paper (glossy and matt photographic paper with a barite layer), and monitoring the effects induced by the Atmospheric Discharge with Runaway Electrons (ADRE) plasma in air atmosphere on the photographic image layers. To evaluate their long-term stability before/after plasma treatment, degradation of black and white gelatin photographic components upon accelerated light aging using Q-SUN was investigated and the photoinduced changes were recorded by FTIR spectroscopy, densitometry and colorimetry. The results obtained demonstrated that the plasma discharge had no significant destructive effect on the photographic image as only negligible changes in the structure of the gelatin were detected due to plasma processing. Consequently, it can be concluded that low-temperature ADRE plasma in air atmosphere has the potential for successful applications in microbial decontamination and purification of damaged gelatin photographs.
\end{abstract}

Keywords: ADRE plasma, colorimetry, densitometry, FTIR spectroscopy, gelatin photography

\section{Introduction}

Photographs are typical of their uniqueness and artistic value. For this reason, there are many efforts to preserve them and prevent their mechanical or microbial damage. Also avoiding various changes caused by the aging process such as color fading or loss of color density has been attempted. (Badea et al., 2008; Haberová, 2018) Because photographs are composed mainly of organic materials such as gelatin or cellulose, they represent suitable culture medium for different fiber fungi as well as for many other microorganisms. (Ioanid et al., 2010; Korachi and Aslan, 2013; Scholtz et al., 2015) Consequently, a great deal of historical photographs is damaged and devaluated as these detrimental processes may lead to their decomposition.

In recent years, more and more information on the applications of low-temperature atmospheric plasma, particularly in the domain of cultural heritage objects including photography, appeared in literature. (Tiňo et al., 2018; Pietrzak et al., 2017) It has been known for a long time that electrical discharges and plasma generated by them have disinfection effect. Plasma is a highly ionized gas composed of a set of neutral particles, free electrons and ions (positive and negative). Ionized gas becomes plasma only at the moment when it begins to exhibit collective behavior and quasi-neutrality. Under this term, we understand approximately identical concentration of positively and negatively charged particles. Plasma can be categorized as low- and high-temperature plasma. (Haberová, 2018; Martišovitš, 2004) In our experiments, lowtemperature air plasma was used.

The advantage of ADRE plasma is its ability to process larger areas at the same time, which makes it suitable for material sterilization. Its discharge has the potential to destroy viruses, bacteria and also fungi (Ioanid et al., 2010; Korachi and Aslan, 2013; Scholtz et al., 2015) and it is applicable also for coarse materials treatment. Another benefit of this type of low-temperature plasma (LTP) is the activation and modification of materials surfaces, curing of deposited layers and formation of required layers on the material surface (hydrophobic, hydrophilic, antibacterial etc.).

The first use of plasma treatment in cultural heritage was in the preservation of historic metallic materials. Plasma is also used in the treatment of daguerreotypes, i.e. photographs on a metal mat (Haberová, 2018; Daniels, 1984; Grieten et al., 2017) and success has also been noted in case of testing its disinfection effect on paper. Also, experiments with plasma treatment of smoke-stained paintings were done to attempt their cleaning or to remove various organic impurities from their surface (Scheider et al., 2013; Vohrer et al., 2001; Banks and Rutledge, 1996). However, all these plasma applications were carried out in vacuum while the use of air plasma 
has been overlooked and its utilization appears in literature only rarely. Direct application of plasma to historical photographs on a paper mat has so far been described only in two publications. (Ioanid et al., 2010; Ioanid et al., 2011) In 2010, positive effect of high-frequency plasma on the decontamination of photographs from fungi as Aspergillus, Cladosporium, Alternaria, Penicillium or Rhizopus was monitored. (Ioanid et al., 2010) In the second case, the effect of low-temperature high-frequency plasma using an $\mathrm{H}_{2} / \mathrm{Ar}$ gas mixture on the surface of two types of historical photographs was determined. In case of gelatin photography, roughness increase and gloss decrease were observed; while in albumin-based photographs, the changes found after plasma treatment were exactly opposite. In both cases, plasma resulted in photobleaching. (Ioanid et al., 2011)

The goal of our work was to determine the effect of plasma discharge on the properties and long-term stability of a photographic image, especially that of low-temperature air plasma on optical properties of photographs and on the structure of gelatin as the main component of the light-sensitive layer. It is necessary to ensure that plasma is not destructive to the photographic image and therefore it is crucial to evaluate all changes of densitometric and colorimetric characteristics as well as FTIR spectra of the prepared samples and their stability depending on the time of plasma treatment.

\section{Materials and Methods}

\section{Materials}

Two types of barytic photographic paper FOMABROM from Foma Bohemia (Hradec Králové, Czech Republic): N 112 (normal, matt), N 111 (normal, glossy) were used.

\section{Preparation of samples}

The above mentioned two types of photographic paper (N 112, N 111) were used for sample preparation (Fig. 1). Exposure by Magnifax 4 (Meopta Přerov, Czech Republic) was done to samples of a photographic image with different optical densities of the $\mathrm{P} 1-\mathrm{P} 8$ fields ( $\mathrm{P} 1$ is the lightest area and $\mathrm{P} 8$ is the darkest area). The exposure time was 0-32 s (with the step change of $4 \mathrm{~s}$ ) thus, the white area was not exposed. Then, the samples were activated, fixed and washed to obtain a permanent photographic image.

Fig. 1. Sample of photographic image with different optical densities P1-P8.

\section{Plasma treatment}

ADRE plasma - Atmospheric Discharge with Runaway Electrons is a low-temperature plasma generated at atmospheric pressure. Fast electrons with the energy of 20-350 keV are formed in the mid-electrode space and behind the anode. Volume stream of the fast electrons is detected behind the anode and is shown as bright light across the area. When the voltage pulse increases two to three times, a change in the radiation between the electrodes can be observed.

The prepared samples were exposed to the ADRE plasma treatment (KAMEA ELECTRONICS, s.r.o., Pieštany, Slovakia) in air atmosphere at $0.6 \mathrm{~J}$, frequency of $2000 \mathrm{~Hz}$ and gas flow rate of $6 \mathrm{~L} \mathrm{~min}^{-1}$. The plasma treatment time was $0.5,1,3,5$ and 10 minutes.

\section{Accelerated light aging in Q-SUN Xenon test chamber (Model Xe-1-S, Q-Lab Corporation, Cleveland, USA) according to ISO 4892-2: 2013 for daylight simulation}

Aging occurred under window glass filters with black body temperature of $65^{\circ} \mathrm{C}$; the radiance at $420 \mathrm{~nm}$ was $1.1 \mathrm{~W} \mathrm{~m}^{-2} ; \mathrm{E}=89 \mathrm{klx}$; incidence of radiation in the range of $300-800 \mathrm{~nm}$ was $494 \mathrm{~W} \mathrm{~m}^{-2}$. Aging was performed for one and for five days.

\section{Densitometry}

Densitometry is a photometric method used to evaluate the proportion of light reflected from a colored surface (reflective densitometry) or passing through this layer (transmission densitometry). (Panák et al., 2000) The result is given in form of optical (photographic) density $D$. In our case, the reflective densitometry measurement was applied. Spectrophotometer SpectroDens (TECHKON, Königstein, Germany), which uses spectral remeasurement according to ISO 5-3/4 and Spectro Connect 2.4, was used to measure the optical density values. The settings were as follows: illumination - D50, standard observer $-2^{\circ}$, polarization filter turned on, white standard absolute. The resulting values were calculated as the average of five measurements.

\section{Colorimetry}

Colorimetry is a photometric method used to measure and characterize color. (Panák et al., 2000) The color space CIELAB serves to evaluate color characteristics of the actual and desired color. Coordinates $L^{*}, a^{*}, b^{*}$ in the CIELAB color space were measured by Spectrophotometer SpectroDens (TECHKON), which uses spectral re-measurement according to ISO 5-3/4 and Spectro Connect 2.4. The settings were as follows: illumination - D50, 
standard observer $-2^{\circ}$, polarization filter off, white standard absolute. The resulting values were calculated as the average of five measurements.

The total color difference $\Delta E_{a b}^{*}$ was calculated using Eq. (1),

$$
\Delta E_{a b}^{*}=\sqrt{\left(\Delta L^{*}\right)^{2}+\left(\Delta a^{*}\right)^{2}+\left(\Delta b^{*}\right)^{2}}
$$

where $\Delta L^{*}, \Delta a^{*}$ and $\Delta b^{*}$ are the differences between relevant values attributed to treated/aged and nontreated/non-aged samples.

\section{Fourier Transform Infrared (FTIR) spectroscopy}

Infrared spectra were measured with an FTIR spectrophotometer (Excalibur Digilab FTS 3000MX, USA) using an ATR adapter with a single reflection diamond crystal at defined positions of fields $\mathrm{P} 1-\mathrm{P} 8$. The measurement range was from 4000 to $700 \mathrm{~cm}^{-1}$; sensitivity -8 ; 30 scans. Reference environment was air. The FTIR spectra were evaluated using the Origin software. In case of samples after plasma treatment, the average of three measurements was used in MS Excel.

\section{Results and Discussion}

\section{Characterization of a photographic image}

Coordinates $L^{*}, a^{*}, b^{*}$ and optical density values obtained during characterization of all the fields for both types of photographic paper are presented in Table 1. With the increasing optical density of the photographic field, the value of $L^{*}$ (lightness) decreases. Since these are achromatic colors, coordinates $a^{*}$ and $b^{*}$ are close to zero. Slightly negative values of $b^{*}$, especially in low optical density fields, are probably due to the addition of optical brighteners to photographic paper and slight differences in the layer thickness.

Figure 2 shows the normalized FTIR spectra (Amide I) obtained for N 112 paper with a photo- graphic image (field $\mathrm{P} 1-\mathrm{P} 8$ ). As it can be seen, the shape of the spectra depends only slightly on the optical density of the photographic image, i.e. on the silver content of the light-sensitive layer $\left(1000-700 \mathrm{~cm}^{-1}\right)$. Typical features in the spectra are protein bands, i.e. Amide I $(A I)$ at $1635 \mathrm{~cm}^{-1}$, Amide II $(A I I)$ at $1557 \mathrm{~cm}^{-1}$, and Amide III $(A I I I)$ between $1200-1350 \mathrm{~cm}^{-1}$. (Singh et al., 2014) The band of Amide A $(A)$ is detectable at $3300 \mathrm{~cm}^{-1}$ and it is usually in form of a doublet with Amide B (B) at $3073 \mathrm{~cm}^{-1}$. (Badea et al., 2008) The shape and intensity of these bands are also affected by vibrations of - $\mathrm{OH}$ groups, which can cause problems in their evaluation. The intensive band at $1060 \mathrm{~cm}^{-1}$ belongs to barium sulfate (barite), which is a component of the barrier layer. (Zhang et al., 2011) In the spectrum of paper N 111 (Fig. 3), this band is not so evident, which may be caused by a thicker light sensitive layer of the glossy paper.

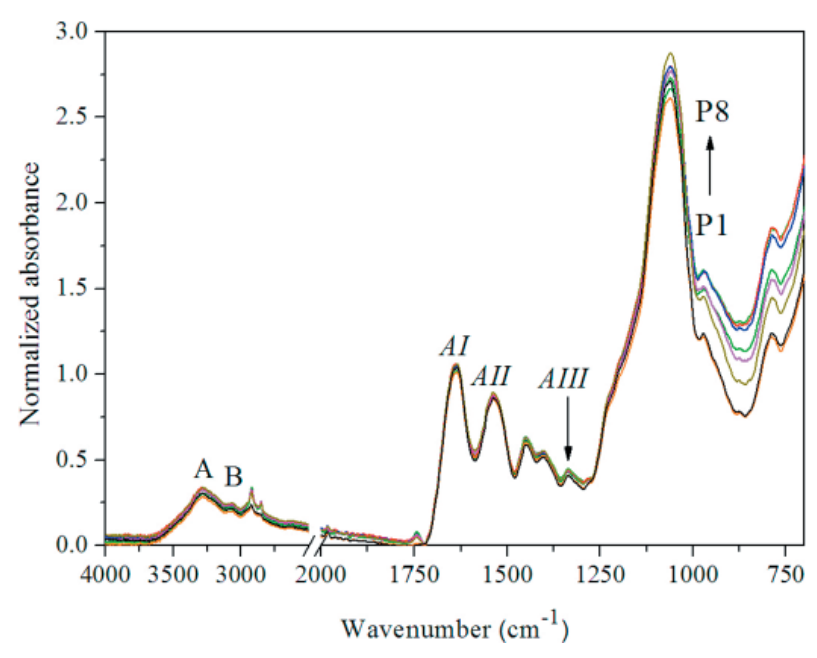

Fig. 2. Normalized FTIR spectrum (Amide I) of photographic image with different optical density $D$ on photo paper N 112, $D$ of individual areas is shown in Table 1 (P1-black, P2 - orange, P3 - dark yellow, P4-purple, P5 - green, P6-blue, P7 - red, P8 - light green).

Tab. 1. Coordinates $L^{*}, a^{*}, b^{*}$ and values of optical density $\mathrm{D}$ for two types of photographic paper: N 112 and $\mathrm{N} 111$ (all values were calculated as the average of five measurements).

\begin{tabular}{|c|c|c|c|c|c|c|c|c|}
\hline \multirow{2}{*}{ Field } & \multicolumn{4}{|c|}{ N 112} & \multicolumn{4}{|c|}{ N 111} \\
\hline & $L^{*}$ & $a^{*}$ & $b^{*}$ & $D$ & $L^{*}$ & $a^{*}$ & $b^{*}$ & $D$ \\
\hline$P 1$ & 95.5 & 0.8 & -2.8 & 0.06 & 96.8 & 0.8 & -2.4 & 0.05 \\
\hline$P 2$ & 91.5 & 0.7 & -2.2 & 0.13 & 95.2 & 0.7 & -2.4 & 0.06 \\
\hline$P 3$ & 74.5 & 0.4 & -0.7 & 0.39 & 85.0 & 0.5 & -1.2 & 0.14 \\
\hline$P 4$ & 55.6 & -0.1 & 0.2 & 0.77 & 65.9 & 0.3 & 0.5 & 0.33 \\
\hline P5 & 44.3 & -0.2 & 0.0 & 1.08 & 49.1 & 0.0 & 1.2 & 0.63 \\
\hline$P 6$ & 38.2 & 0.0 & -0.3 & 1.32 & 38.1 & 0.1 & 1.3 & 0.93 \\
\hline$P 7$ & 34.9 & -0.1 & -0.5 & 1.49 & 31.3 & 0.3 & 1.2 & 1.15 \\
\hline P8 & 33 & -0.1 & -0.6 & 1.57 & 26.6 & 0.6 & 0.9 & 1.31 \\
\hline
\end{tabular}




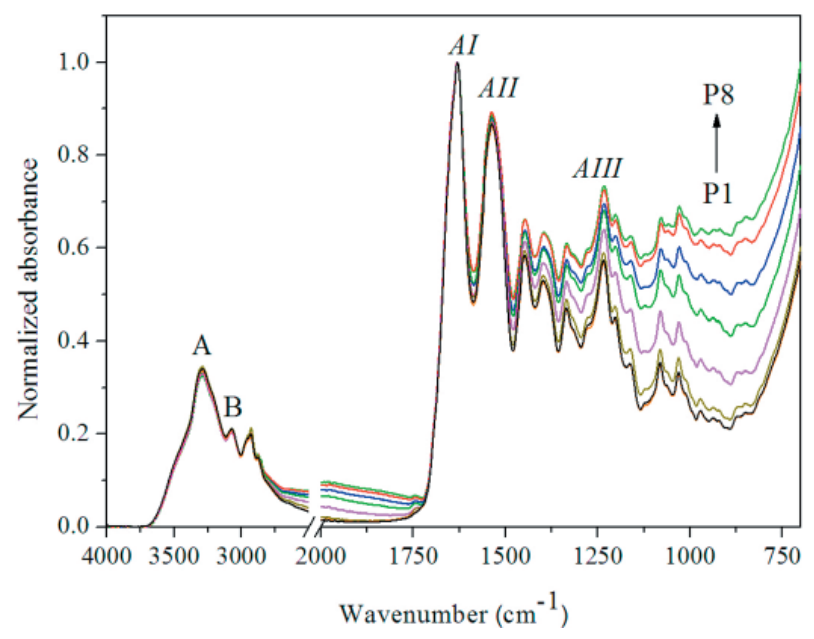

Fig. 3. Normalized FTIR spectrum (Amide I) of photographic image with different optical density $D$ on photo paper N 111, $D$ of individual areas is shown in Table 1 ( $P 1$ - black, $P 2$ - orange, P3-dark yellow, P4-purple, P5-green, P6-blue, P7 - red, P8-light green).

\section{Influence of plasma treatment on photographic image}

As a result of plasma treatment, a slight decrease in the optical density across the photographic image on N 112 matt paper can be observed, except for the P1 field (white), while the most significant decrease was determined for the dark fields P5-P8, where it reached the value of 0.12 after ten minutes of plasma treatment. On the other hand, optical density increase was observed on gloss paper N 111 especially in the gray fields of the photographic image (in P5, optical density increased by 0.15) due to plasma treatment. Based on measured $L^{*}, a^{*}, b^{*}$ coordinates, the values of $\Delta E_{a b}^{*}$ were calculated over the whole range of the photographic image (fields $\mathrm{P} 1-\mathrm{P} 8$ ). The evaluated $\Delta E_{a b}^{*}$ were minimal ( 1.3), not exceeding the declared limit of 2.0, which corresponds to the minimum difference that is not perceptible even in direct comparison of the samples. (Panák et al., 2000)

FTIR spectra at individual sites of the photographic image were measured and compared in order to identify changes caused by plasma treatment. In FTIR spectra of photographic image on N 112 paper after plasma exposure, minor changes in the range of $1650-1250 \mathrm{~cm}^{-1}$ were detected. The absorbance of band AII decreased and the absorbance of band AIII increased. These changes are similar in all fields (independent of optical density of the photographic image) and indicate possible hydrolytic damage of gelatin. Simultaneously, certain changes in the area typical of $\mathrm{CH}_{\mathrm{x}}$ groups (2800-2900 $\left.\mathrm{cm}^{-1}\right)$ was observed. As an illustrative example, FTIR spectra of the photographic image of field P1 and P8 for N 112 photographic paper are shown in Fig. 4.

Absorbance of individual amide bands was evaluated from the FTIR spectra using the baseline method and the calculated ratios of Amide I and Amide II $(A I / A I I)$; their increase indicates the degree of hydrolytic damage of gelatin and is summarized in Fig. 5. From the given results it appears that on all the fields of the photographic image of both paper types, this ratio is slightly increased due to plasma treatment, revealing a little higher increment for $\mathrm{N} 112$ paper.
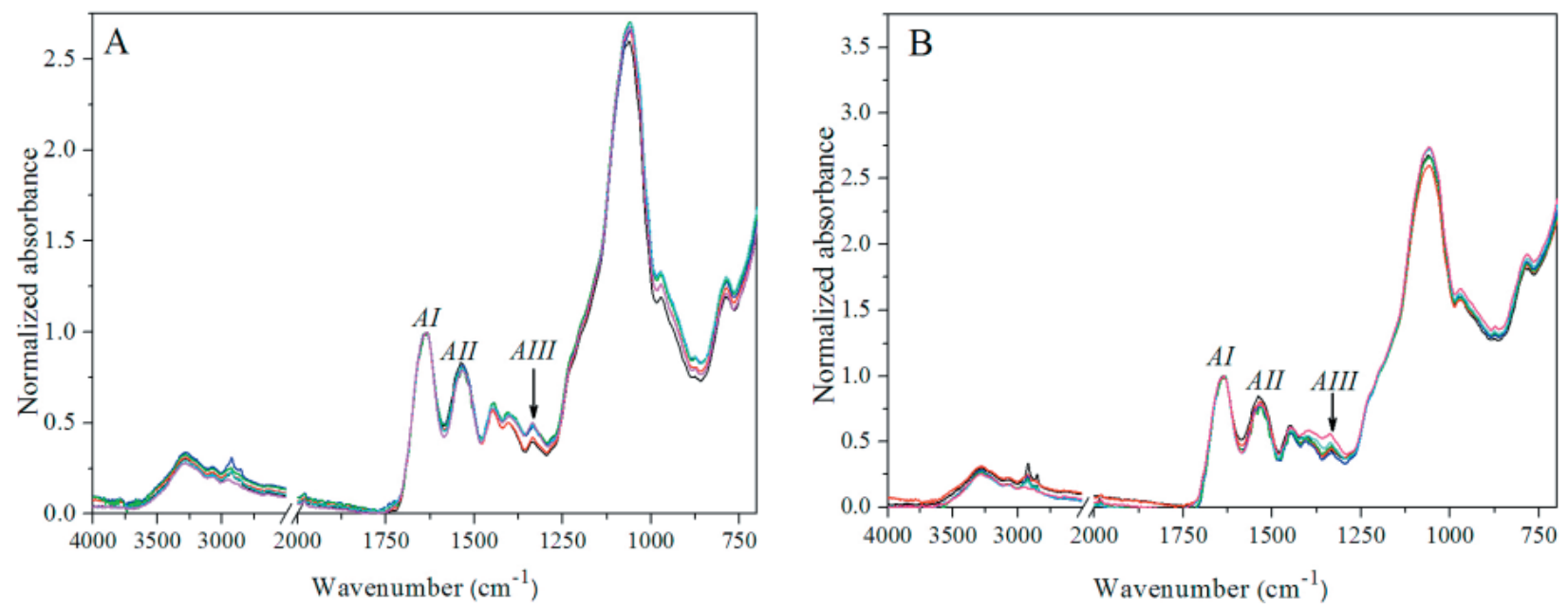

Fig. 4. Normalized FTIR spectra (Amide I) of photographic images of fields P1(A) and P8 (B) on photographic paper N 112 and their changes after plasma treatment (untreated samples - black line and samples after 30 seconds of plasma treatment - red line, 1 min - blue line, 3 min - green line, 5 min-azure line and 10 min - pink line). 


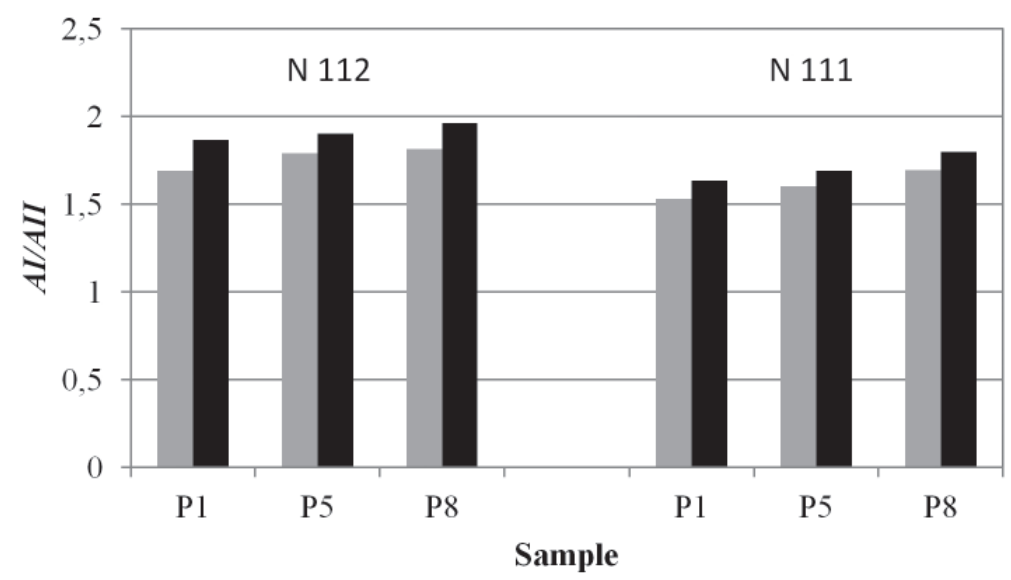

Fig. 5. Graphical representation of calculated ratios of Amide I (AI)/Amide II (AII) (untreated samples - gray, samples after 10 min of plasma treatment - black) for the selected fields P1, P5 and P8.
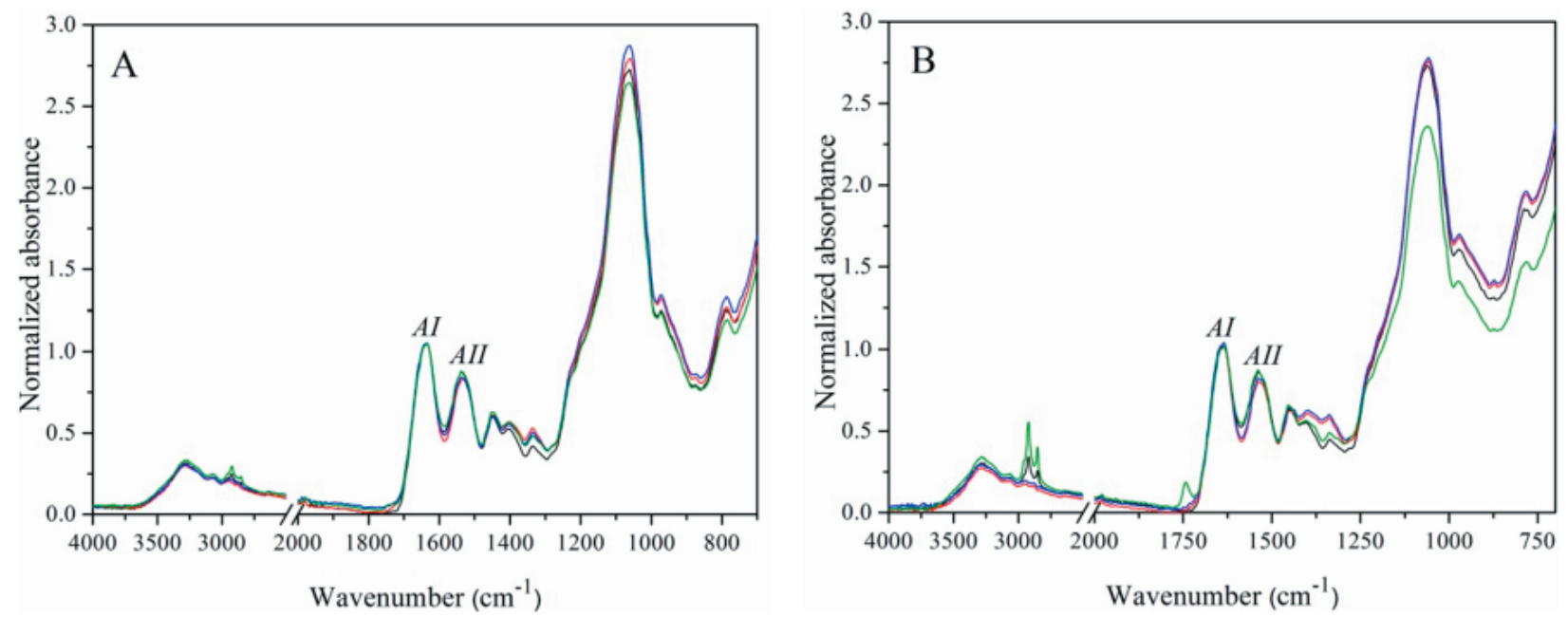

Fig. 6. Normalized FTIR spectra (Amide I) of photographic images of fields P1(A) and P8 (B) on N 112 photographic paper (untreated sample - black line, samples after plasma treatment, spectra of which were measured immediately after plasma treatment - red line, two days after plasma treatment - blue line and seven days after plasma treatment - green line).

Also changes occurring in the samples immediately after plasma treatment and after steady-state (2-7 days) storage of the plasma-treated samples under laboratory conditions in dark were monitored (Fig. 6). While the samples with low optical density of the photographic image (field P1) returned to their original values as they were before plasma treatment, for samples with high optical density (P8), a band appeared at $1730 \mathrm{~cm}^{-1}$ (oxidation products), accompanied with changes in the region of the $\mathrm{CH}_{\mathrm{x}}$ bands $\left(2800-2900 \mathrm{~cm}^{-1}\right)$.

\section{Accelerated aging in Q-sun chamber}

To assess the effect of plasma treatment on longterm photostability, the prepared samples were aged in a Q-sun chamber. Untreated and also samples after a 10-min plasma treatment were aged using the total exposure of 120 hours representing the total energy of $475 \mathrm{~kJ} / \mathrm{m}^{2}$.

A change was noticed after the accelerated light aging of untreated samples. On photographic paper $\mathrm{N} 112$, a decrease in optical densities, especially in the darker fields (P6-P8) was observed, while on glossy paper $\mathrm{N} 111$, the optical density increased mainly in the gray fields (P4-P6). It is interesting that in case of plasma-treated samples, light aging did not change the optical density.

$L^{*}, a^{*}$ and $b^{*}$ coordinates of untreated samples and of samples after plasma treatment were compared. Untreated paper $\mathrm{N} \mathrm{112,} \mathrm{due} \mathrm{to} \mathrm{accelerated} \mathrm{aging} \mathrm{of} \mathrm{the}$ photographic image, showed changes in the $L^{*}, a^{*}$ and $b^{*}$ values depending on the initial optical density of the photographic fields. While the $L^{*}$ coordinate (lightness) for the low optical density fields (P1-P3) 

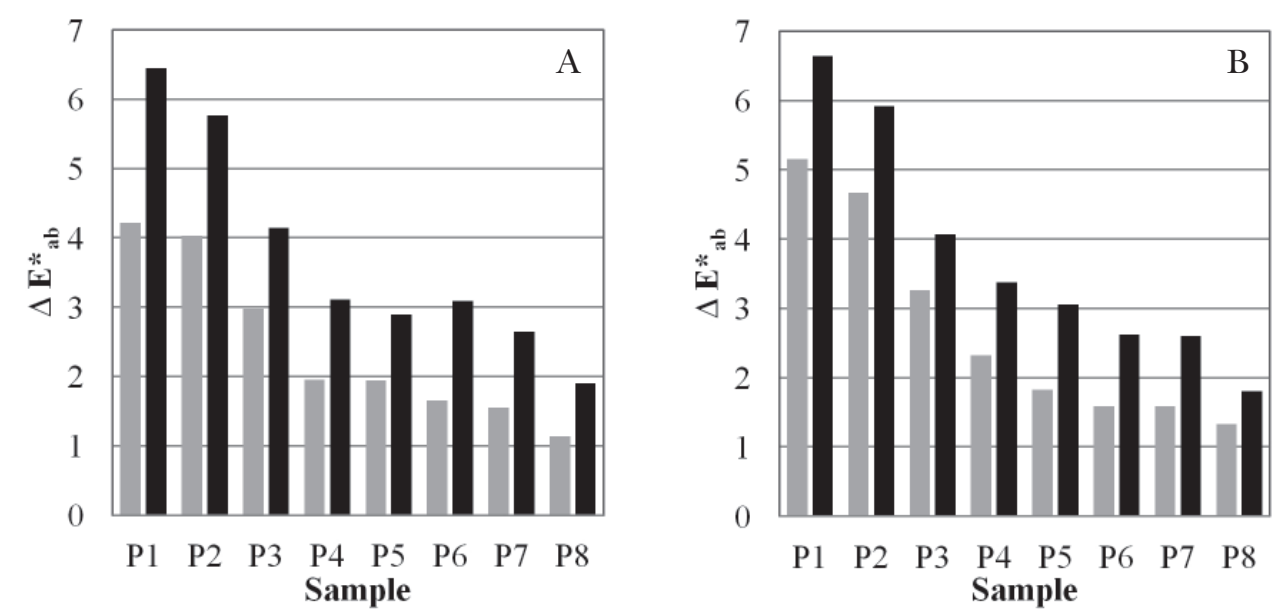

Fig. 7. Total color difference $\Delta E_{a b}^{*}$ after aging for one day (gray columns) and for five days (black columns) for untreated (A) and plasma treated photographic paper (B) N 112.
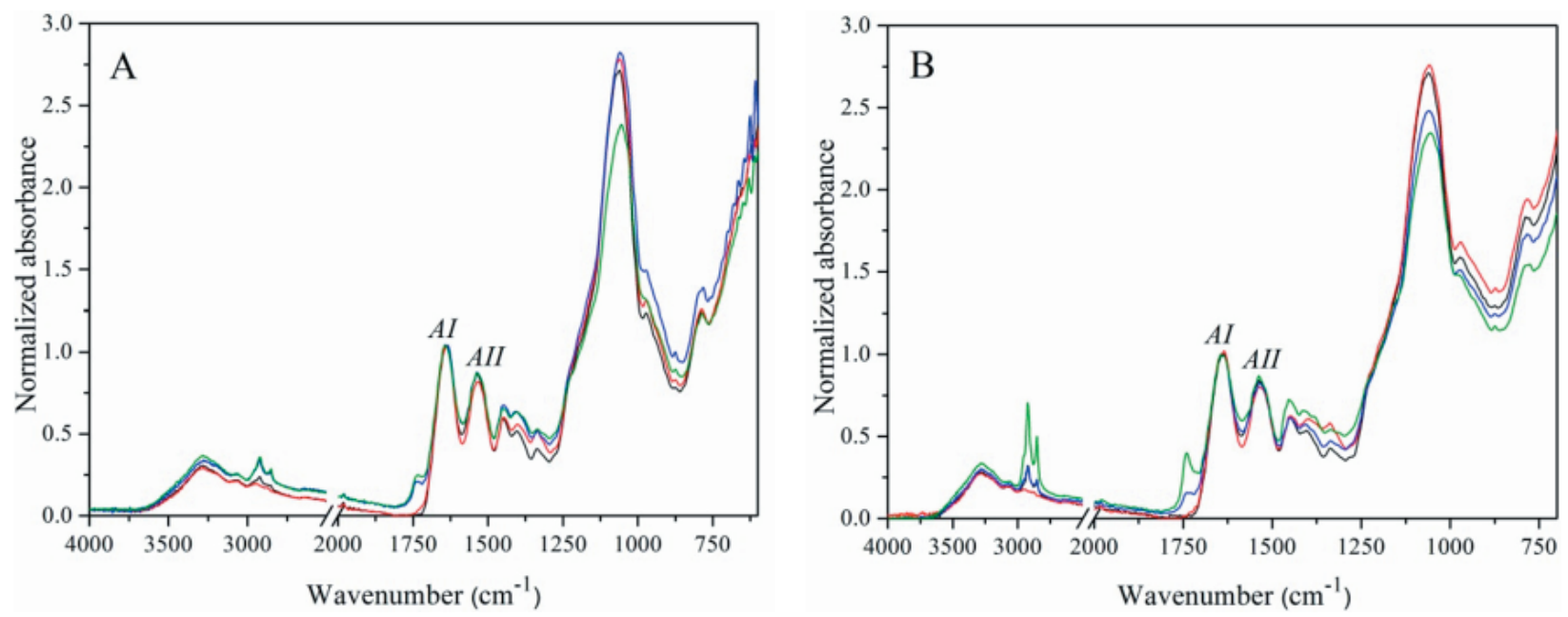

Fig. 8. Normalized FTIR spectra (Amide I) of photographic images of fields P1 (A) and P8 (B) for photographic paper N 112 (untreated sample - black line, sample after plasma treatment for $10 \mathrm{~min}$ - red line, untreated sample aged in Q-SUN for five days - blue line, sample after plasma treatment for $10 \mathrm{~min}$ and then aged in Q-SUN for five days - green line.

was almost identical to control values, slight increase associated with image bleaching was detected in the dark samples. Differences in coordinate $a^{*}$ were small, and the most significant changes were observed for coordinate $b^{*}$ for low optical density fields (e.g. for field $\mathrm{P} 1, \Delta b^{*}=6.3$ ). With the optical density increase of the photographic field, the increase in $\Delta b^{*}$ was less significant. The total color difference $\left(\Delta E_{a b}^{*}\right)$ correlates well with the major changes of the $b^{*}$ coordinate. As it can be seen in Fig. 7 , for the low optical density fields (P1-P3) $\Delta E_{a b}^{*}$ after five days of accelerated aging is in the range of 4-6.5, which represents changes observable only in direct comparison. (Panák et al., 2000) Almost identical differences also occurred in plasma-treated samples subjected to accelerated aging. It can be assumed that the use of ADRE plasma does not promote light aging of photographic image.
FTIR spectra shown in Fig. 8 show that during accelerated light aging in the Q-SUN chamber, an increase in absorbance coupled with the formation of a band at $1730 \mathrm{~cm}^{-1}$ occurs in all samples (nontreated/plasma-treated samples). This band can be attributed to oxidative products generated in materials upon accelerated light aging, and its more pronounced appearance in paper N 112 may reflect the different properties of the studied papers. Whereas matt paper (N 112) absorbs a significant amount of radiation, glossy paper (N 111) reflects this radiation. In case of matt paper, photoinduced formation of oxidation products is more significant. The extent of oxidative degradation is closely related to the optical density of the photographic image, therefore it is most pronounced in the darkest fields (P8) of both papers. In case of oxidation products generated upon accelerated light aging, 
the promoting effect of plasma processing can be seen in comparison to the non-treated samples (green line in Fig. 8B).

\section{Conclusions}

Colorimetry, densitometry and FTIR spectroscopy were applied for the characterization of optical and spectral properties of a photographic image with different optical densities prepared on two types of photographic paper FOMABROM (matt and glossy) with a barite layer. Also, the changes observed after the air plasma treatment and accelerated aging in a Q-SUN chamber were monitored.

Using densitometry and colorimetry, minimal changes in optical density and colorimetric coordinates due to air ADRE plasma treatment were observed and the plasma processing did not damage the photographic image. Using FTIR spectroscopy, the changes in the gelatin structure in the image layer were evaluated. It was found that, due to plasma activity, the Amid I/Amid II ratio slightly increased, indicating possible hydrolytic damage of gelatin and, in some cases, simultaneously forming a smaller amount of oxidation products. Under dark storage, this phenomenon is permanent for the darker photographic fields; however, plasmainduced changes disappeared for the low density fields.

Upon accelerated light aging of non-treated/ plasma-treated samples in the Q-SUN chamber, no significant difference in optical density and in the stability of the photographic image were found. The only negative phenomenon detected was a slightly increased production of oxidation products in case of accelerated light aging of plasma-treated samples in the fields with the highest optical density of the photographic image.

The fact that low-temperature ADRE plasma does not have a negative effect on the photographic image and its long-term stability in air atmosphere and that plasma-treatment induces no significant changes in the gelatin layer support, this technique seems to be a perspective tool for microbial decontamination and cleaning of photographs.

\section{Acknowledgements}

The authors thank the Slowak Grant Agencies APVV (Project No. 15-0460 SK) and VEGA (Project No. 1/0602/19) for financial support.

\section{References}

Badea E, Miu L, Budrugeac P, Giurginca M, Masic A, Badea N, Della Gatta G (2008) Journal of Thermal Analysis and Calorimetry 91: 17-27.

Banks BA, Rutledge SK (1996) US Patent 5560781.

Daniels V (1984) Studies in Conservation 26: 45-49.

Grieten E, Schalm O, Tack P, Bauters S, Storme P, Gauquelin N, Caen J, Patelli A, Vincze L, Schryvers D (2017) Journal of Cultural Heritage 28: 56-64.

Haberová K (2018) Written work on the dissertation test, STU Bratislava.

Ioanid EG, Rusu D, Dunca S, Tanase C (2010) Ann Microbiol 60: 355-361.

Ioanid EG, Ioanid A, Rusu DE, Doroftei F (2011) Journal of Cultural Heritage 12: 296-317.

Korachi M, Aslan N (2013) In: Méndez-Vilas (Ed.), Microbial pathogens and strategies for combating them: science, technology and education, Vol. 1: 453-459.

Martišovitš V (2004) Základy fyziky plazmy, Univerzita Komenského, Bratislava.

Panák J, Čeppan M, Dvonka V, Karpinský L, Kordoš P, Mikula M, Jakucewicz S (2008) Polygrafické minimum, TypoSet, Bratislava.

Pietrzak K, Puchalski M, Otlewska A, Wrzosek H, Guiamet P, Piotrowska M, Gutarowska B (2017) Journal of Cultural Heritage 24: 69-77.

Scheider JP, Vepřek S (2013) Studies in Conservation, 31: 29-37.

Scholtz V, Pazlarova J, Souskova H, Khun J, Julak J (2015) Biotechnology Advances 33: 1108-1119.

Singh BR, DeOliveira DB, Fu F, Fuller MP (1993) Proc. SPIE 1890, Biomolecular Spectroscopy III, Los Angeles.

Tiňo R, Vizárová K, Krčma F (2018) In: Lazzara G, Fakrullin R (Ed) Nanotechnologies and nanomaterials for diagnostic, conservation and restoration of cultural heritage, (pp 239-275). Elsevier Publishing.

Vohrer U, Trick I, Bernhardt J, Oehr C, Brunner H (2001) Surface and Coatings Technology 142-144: 1069-1073.

Zhang M, Zhang B, Li X, Yin Z, Guo X (2011) Applied Surface Science 258: 24-29. 